\title{
NOISE ROBUST BLIND SYSTEM IDENTIFICATION ALGORITHMS BASED ON A RAYLEIGH QUOTIENT COST FUNCTION
}

\author{
Mathieu Hu*, Simon Doclo ${ }^{\dagger}$, Dushyant Sharma ${ }^{\ddagger}$, Mike Brookes $^{*}$, Patrick Naylor* \\ * Department of Electrical and Electronic Engineering, Imperial College London, UK \\ $\dagger$ Department of Medical Physics and Acoustics and Cluster of Excellence Hearing4All, \\ University of Oldenburg, Oldenburg, Germany \\ $\ddagger$ Voicemail-To-Text Research, Nuance Communications Inc. Marlow, UK
}

\begin{abstract}
An important prerequisite for acoustic multi-channel equalization for speech dereverberation involves the identification of the acoustic channels between the source and the microphones. Blind System Identification (BSI) algorithms based on cross-relation error minimization are known to misconverge in the presence of noise. Although algorithms have been proposed in the literature to improve robustness to noise, the estimated room impulse responses are usually constrained to have a flat magnitude spectrum. In this paper, noise robust algorithms based on a Rayleigh quotient cost function are proposed. Unlike the traditional algorithms, the estimated impulse responses are not always forced to have unit norm. Experimental results using simulated room impulse responses and several SNRs show that one of the proposed algorithms outperforms competing algorithms in terms of normalized projection misalignment.
\end{abstract}

Index Terms - Blind System Identification, Rayleigh quotient, Least Mean Squares (LMS), noise robustness, dereverberation

\section{INTRODUCTION}

Capturing a sound source within an enclosed environment with microphones placed at a distance from the sound source typically results in recordings that are corrupted by reverberation, i.e. acoustic reflections against walls and surfaces within the enclosure. While reverberation may be desirable in music, it may degrade the quality and the intelligibility of speech [1], especially in the presence of additive noise, so that dereverberation is needed.

Since the recorded microphone signals consist of superimposed delayed and attenuated copies of different sound events, each microphone signal can be modeled as a source signal filtered by an acoustic channel represented by a Room Impulse Response (RIR). Consequently, dereverberation can

* The research leading to these results has received funding from the European Union's Seventh Framework Programme (FP7/2007-2013) under grant agreement n ITN-GA-2012-316969. potentially be achieved by first blindly estimating the RIRs and then equalizing the reverberant signal. Perfect dereverberation can be achieved [2] provided that the estimates of the RIRs are perfect. Algorithms for designing equalizers that are more robust to estimation errors have been proposed in [3] or [4] for example.

In the recent literature, different adaptive algorithms for BSI of RIRs have been proposed [5]-9], which are based on minimizing the cross-relation error cost function. However, it has been reported that adaptive BSI algorithms such as the Multi-Channel Least Mean Square (MCLMS) [5] or the Normalized Multichannel Frequency Domain Least Mean Squares (NMCFLMS) [6] misconverge in the presence of additive noise [10]. To improve robustness to noise, the method proposed in [11] assumes that the delay and magnitude of the direct path are known for each RIR. In [9] and [8], a spectral flatness constraint of the magnitude spectrum of the estimated RIRs is added to the cost function. Although improvements have been observed, the spectral flatness constraint may be too restrictive as the magnitude spectrum of a RIR is not necessarily flat. Accordingly, the Robust NMCFLMS (RNMCFLMS) [9] may lead to an estimation which is a trade-off between minimizing the cross-relation error and satisfying the constraint.

In this paper, novel adaptive BSI algorithms relying on a Rayleigh quotient cost function are proposed. In Section 2 , an overview of BSI from an algebraic point of view is presented. The MCLMS and NMCFLMS algorithms are briefly reviewed in Section 3. Several novel variants of the MCLMS algorithm are presented in Section 4 Section 5 shows the results of simulations, comparing the performance of the proposed algorithms against the MCLMS, the NMCFLMS and the RNMCFLMS algorithms.

\section{BSI BACKGROUND}

\subsection{Signal model and assumptions}

We consider an acoustic system with $M$ microphones and a single sound source. The noisy and reverberant signal $y_{m}(n)$ 
at the $m^{t h}$ microphone, $m \in\{1, \ldots, M\}$, can be written as:

$$
\begin{gathered}
x_{m}(n)=h_{m} * s(n), \\
y_{m}(n)=x_{m}(n)+\nu_{m}(n)
\end{gathered}
$$

where $s(n)$ is the source signal, $h_{m}$ the RIR between the source and the $m^{t h}$ microphone, which is assumed to be a fixed Finite Impulse Response (FIR)-filter with length $L$, $\nu_{m}(n)$ the additive noise component at the $m^{t h}$ microphone and $x_{m}(n)$ the reverberant noiseless signal at the $m^{\text {th }}$ microphone.

With reference to the identifiability conditions [13], we assume that the source has a full-rank covariance matrix and the RIRs have no common zeros.

\subsection{Cross-relation error}

The MCLMS and NMCFLMS algorithms [5, 6] exploit the Single-Input-Multiple-Output (SIMO) structure of the problem in (1) to estimate the RIRs.

The cross-relation error $\chi(n)$ is defined as:

$$
\begin{array}{r}
\chi(n)=\sum_{i=1}^{M-1} \sum_{j=i+1}^{M} e_{i j}^{2}(n), \\
e_{i j}(n)=\boldsymbol{y}_{i}^{T}(n) \hat{\boldsymbol{h}}_{j}(n)-\boldsymbol{y}_{j}^{T}(n) \hat{\boldsymbol{h}}_{i}(n)
\end{array}
$$

where $\boldsymbol{y}_{i}(n)=\left[\begin{array}{llll}y_{i}(n) & y_{i}(n-1) & \ldots & y(n-L+1)\end{array}\right]^{T}$ and $\hat{\boldsymbol{h}}_{i}(n)=\left[\begin{array}{llll}\hat{h}_{i}(0) & \hat{h}_{i}(1) & \ldots & \hat{h}_{i}(L-1)\end{array}\right]^{T}$ is an estimate of $\boldsymbol{h}_{i}$. Estimates $\hat{\boldsymbol{h}}(n)=\left[\hat{\boldsymbol{h}}_{1}^{T}(n), \hat{\boldsymbol{h}}_{2}^{T}(n), \ldots, \hat{\boldsymbol{h}}_{M}^{T}(n)\right]^{T}$ of the stacked RIRs $\boldsymbol{h}$ are obtained by minimizing the expectation of $\chi(n)$, subject to the constraint $\hat{\boldsymbol{h}} \neq \mathbf{0}_{M L \times 1}$, where $\mathbf{0}_{M L \times 1}$ is the null vector of size $M L$.

This minimization problem is equivalent to computing the eigenvector corresponding to the smallest eigenvalue of the expectation of the cross-relation matrix $\hat{\boldsymbol{R}}(n)[5]$ given by

$$
\hat{\boldsymbol{R}}=\left[\begin{array}{cccc}
\sum_{i=2}^{M} \hat{\boldsymbol{R}}_{\boldsymbol{y}_{i} \boldsymbol{y}_{i}} & -\hat{\boldsymbol{R}}_{\boldsymbol{y}_{2} \boldsymbol{y}_{1}} & \ldots & -\hat{\boldsymbol{R}}_{\boldsymbol{y}_{M}} \boldsymbol{y}_{1} \\
-\hat{\boldsymbol{R}}_{\boldsymbol{y}_{1} \boldsymbol{y}_{2}} & \sum_{i \neq 2}^{M} \hat{\boldsymbol{R}}_{\boldsymbol{y}_{i} \boldsymbol{y}_{i}} & \ldots & -\hat{\boldsymbol{R}}_{\boldsymbol{y}_{M} \boldsymbol{y}_{2}} \\
\vdots & \vdots & \ddots & \vdots \\
-\hat{\boldsymbol{R}}_{\boldsymbol{y}_{1} \boldsymbol{y}_{M}} & -\hat{\boldsymbol{R}}_{\boldsymbol{y}_{1} \boldsymbol{y}_{M}} & \cdots & \sum_{i=1}^{M-1} \hat{\boldsymbol{R}}_{\boldsymbol{y}_{i} \boldsymbol{y}_{i}}
\end{array}\right]
$$

where $\hat{\boldsymbol{R}}_{\boldsymbol{y}_{i} \boldsymbol{y}_{j}}=\boldsymbol{y}_{i} \boldsymbol{y}_{j}^{T}$ and the time index $n$ has been dropped. We will refer to the expectation of $\hat{\boldsymbol{R}}(n)$ as $\boldsymbol{R}$.

Noticing that $e_{i j}^{2}(n)=e_{j i}^{2}(n)$ and $e_{i i}(n)=0$, the crossrelation error $\chi(n)$ is equal to $\frac{1}{2} \sum_{i=1}^{M} \sum_{j=1}^{M} e_{i j}^{2}(n)$. Expanding $e_{i j}^{2}(n)$ using 4 , gives

$$
\begin{aligned}
\chi(n) & =\sum_{i=1}^{M} \sum_{j=1}^{M} \hat{\boldsymbol{h}}_{j}^{T} \hat{\boldsymbol{R}}_{\boldsymbol{y}_{i} \boldsymbol{y}_{i}} \hat{\boldsymbol{h}}_{j}^{T}-\hat{\boldsymbol{h}}_{i}^{T} \hat{\boldsymbol{R}}_{\boldsymbol{y}_{i} \boldsymbol{y}_{j}} \hat{\boldsymbol{h}}_{j}^{T}, \\
\Rightarrow \chi(n) & =\hat{\boldsymbol{h}}^{T}(n) \hat{\boldsymbol{R}}(n) \hat{\boldsymbol{h}}(n) .
\end{aligned}
$$

\subsection{Effect of Additive noise}

In the absence of additive noise, the null space of the matrix $\boldsymbol{R}$ is of dimension 1 and is spanned by the stacked RIRs $\boldsymbol{h}$ [13]. However, in the presence of noise, this property does not hold anymore as $\boldsymbol{R}$ is the sum of the expectation of the cross-relation matrix of the noiseless reverberant signal $\boldsymbol{R}_{\boldsymbol{x} \boldsymbol{x}}$ and of the noise component $\boldsymbol{R}_{\boldsymbol{\nu} \boldsymbol{\nu}}$, assuming that $\nu_{m}(n)$ and $x_{m}(n)$ are uncorrelated [14].

In the case of additive spatially white Gaussian noise, $\boldsymbol{R}_{\nu \nu}$ is proportional to the identity matrix of size $M L \times M L$ and the eigenvalues of $\boldsymbol{R}_{\boldsymbol{x} \boldsymbol{x}}$ are shifted by the noise power, while the associated eigenvectors remain unchanged.

\section{EXISTING ALGORITHMS}

In this section, we analyze the cost function employed in two existing algorithms that adaptively solve the minimization problem in (3).

\subsection{MCLMS}

It can be shown that the MCLMS algorithm proposed in [5] solves the eigenvector estimation problem (5) by minimizing the Rayleigh quotient (RQ) cost function $J_{\mathrm{RQ}}(n)$ given by

$$
J_{\mathrm{RQ}}(n)=\frac{\hat{\boldsymbol{h}}^{T}(n) \hat{\boldsymbol{R}}(n) \hat{\boldsymbol{h}}(n)}{\hat{\boldsymbol{h}}^{T}(n) \hat{\boldsymbol{h}}(n)},
$$

using an LMS-type adaptive algorithm.

In the MCLMS algorithm, the estimated stacked RIR vector $\hat{\boldsymbol{h}}(n)$ is forced to have unit-norm after each iteration. The update equation is given by

$$
\begin{aligned}
\hat{\boldsymbol{h}}(n+1) & =\frac{\hat{\boldsymbol{h}}(n)-\mu \nabla J_{\mathrm{RQ}}(n)}{\left\|\hat{\boldsymbol{h}}(n)-\mu \nabla J_{\mathrm{RQ}}(n)\right\|_{2}} \\
\nabla J_{\mathrm{RQ}}(n) & =\frac{2}{\hat{\boldsymbol{h}}^{T}(n) \hat{\boldsymbol{h}}(n)}\{\hat{\boldsymbol{R}}(n) \hat{\boldsymbol{h}}(n)-\chi(n) \hat{\boldsymbol{h}}(n)\}
\end{aligned}
$$

where $\nabla J_{\mathrm{RQ}}(n)$ is the gradient of the RQ cost function $J_{R Q}(n)$ and $\mu$ is a small constant positive step-size.

\subsection{NMCFLMS}

In [6], the implementation is performed in the frequency domain to efficiently compute convolutions. Following the same strategy as above to reformulate the cross-relation error into a quadratic product, it can be shown that the instantaneous cost function, the expectation of which is minimized, is given by

$$
J_{\text {NMCFLMS }}(b)=\underline{\hat{\boldsymbol{h}}}^{H}(b) \underline{\hat{\boldsymbol{R}}}(b) \underline{\hat{\boldsymbol{h}}}(b)
$$

where (.) denotes a complex-valued quantity, the superscript $H$ denotes the conjugate transpose operator, $\underline{\hat{\boldsymbol{h}}}(b)=\boldsymbol{I}_{M \times M} \otimes$ 
$\underline{\boldsymbol{F}}_{L \times L} \hat{\boldsymbol{h}}(b)$ and $\underline{\hat{\boldsymbol{R}}}(b)=\left(\boldsymbol{I}_{M \times M} \otimes \boldsymbol{F}_{L \times L}\right) \hat{\boldsymbol{R}}(b)\left(\boldsymbol{I}_{M \times M} \otimes\right.$ $\left.\boldsymbol{F}_{L \times L}^{-1}\right)$. The matrices $\boldsymbol{I}_{M \times M}, \boldsymbol{F}_{L \times L}$ and $\hat{\boldsymbol{R}}(b)$ respectively correspond to the identity matrix of size $M \times M$, the discrete Fourier transform matrix of size $L \times L$ and the crossrelation matrix $\hat{\boldsymbol{R}}(n)$ averaged over $L$ samples. The $\otimes$ operator denotes the Kronecker product and $b$ corresponds to a time-frame block index.

The NMCFLMS algorithm implements a modified block LMS, the processing of which is performed in the frequency domain. However, note that the quadratic cost function in (11) significantly differs from the RQ cost function $J_{\mathrm{RQ}}(n)$ in (8) as the denominator is equal to 1 .

To increase the convergence rate, a preconditioner $\underline{\boldsymbol{P}}(b)$ approximating the inverse of the Hessian of $J_{\text {NMCFLMS }}(b)$ is used. The update equation is therefore:

$$
\underline{\hat{\boldsymbol{h}}}(b+1)=\frac{\underline{\hat{\boldsymbol{h}}}(b)-\mu \underline{\boldsymbol{P}}(b) \underline{\nabla} J_{\mathrm{NMCFLMS}}(b)}{\left\|\underline{\hat{\boldsymbol{h}}}(b)-\mu \underline{\boldsymbol{P}}(b) \underline{\nabla} J_{\mathrm{NMCFLMS}}(b)\right\|_{2}}
$$

where $\nabla J_{\text {NMCFLMS }}=\underline{\hat{\boldsymbol{R}}}(b) \underline{\hat{\boldsymbol{h}}}(b)$ and the expression for $\underline{\boldsymbol{P}}(b)$ can be found in [6].

\section{PROPOSED ALGORITHMS}

In this section, we propose novel adaptive BSI algorithms that are based on the RQ cost function in (8), by using different approximations for $\boldsymbol{R}$ with or without imposing a unit-norm constraint.

In the update equation of an LMS-based procedure, the direction of the descent steers the adaption towards a point minimizing the cost function. In the case where $\boldsymbol{R}$ is approximated by its instantaneous value $\hat{\boldsymbol{R}}(n)$, as e.g. in the MCLMS algorithm, there exists a high-dimensional subspace which spans vectors nullifying $J_{\mathrm{RQ}}(n)$. When no noise is present, the stacked RIRs $\boldsymbol{h}$ lie in the null space of $\hat{\boldsymbol{R}}(n)$ for all $n$. However, this is not guaranteed in the presence of noise.

Moreover, in the presence of noise, the cost function in (11) achieves its minimum only at the null vector such that the normalization in (12) is required to avoid this trivial solution. On the other hand, the RQ cost function in $(8)$ implicitly excludes the null vector so that the normalization in $(9)$ is in principle not necessary.

We therefore propose a class of adaptive algorithms based on the RQ cost function in (8). The different variants differ in the approximation of $\boldsymbol{R}$ (sample-based vs. block-based) as well as the presence or absence of a unit-norm constraint, giving rise to the Sample-based RQ (SURQ), the Block-based Normalized RQ (BNRQ) and the Block-based Unconstrained RQ (BURQ) algorithms (see Table 1). The corresponding update equations are respectively given by

$$
\begin{aligned}
\hat{\boldsymbol{h}}(n+1) & =\hat{\boldsymbol{h}}(n)-\mu \nabla J_{\mathrm{RQ}}(n), \\
\hat{\boldsymbol{h}}(b+1) & =\frac{\hat{\boldsymbol{h}}(b)-\mu \nabla J_{\mathrm{RQ}}(b)}{\left\|\hat{\boldsymbol{h}}(b)-\mu \nabla J_{\mathrm{RQ}}(b)\right\|_{2}}, \\
\hat{\boldsymbol{h}}(b+1) & =\hat{\boldsymbol{h}}(b)-\mu \nabla J_{\mathrm{RQ}}(b)
\end{aligned}
$$

where

$$
\nabla J_{\mathrm{RQ}}(b)=\frac{2}{\hat{\boldsymbol{h}}^{T}(b) \hat{\boldsymbol{h}}(b)}\{\hat{\boldsymbol{R}}(b) \hat{\boldsymbol{h}}(b)-\chi(b) \hat{\boldsymbol{h}}(b)\},
$$

with $\hat{\boldsymbol{R}}(b)=\frac{1}{B} \sum_{l=0}^{B-1} \hat{\boldsymbol{R}}\left(b n_{\text {of } f}+l\right), B$ and $n_{\text {off }}$ respectively representing the block size and a sliding offset and $\chi(b)=\hat{\boldsymbol{h}}^{T}(b) \hat{\boldsymbol{R}}(b) \hat{\boldsymbol{h}}(b)$.

\begin{tabular}{|c|c|c|}
\hline Algorithm & Approximation of $\boldsymbol{R}$ & Additional step \\
\hline MCLMS $\sqrt{9})$ & Sample-based & Forced unit-norm \\
SURQ $\sqrt{13}$ & Sample-based & None \\
BNRQ $\sqrt{14}$ & Block-based & Forced unit-norm \\
BURQ $\sqrt{15}$ & Block-based & None \\
\hline
\end{tabular}

Table 1: Rayleigh quotient based algorithms for BSI

\section{SIMULATIONS}

In this section, the performance of the proposed RQ-based algorithms is investigated and compared with the existing MCLMS, NMCFLMS and RNMCFLMS algorithms. In particular, the robustness to additive spatially white Gaussian noise and the convergence rate are evaluated.

\subsection{Setup}

The algorithms were evaluated for two RIR lengths ( $L=$ 256 and $L=500$ ), two different numbers of microphones, ( $M=2$ and $M=5$ ), and two values of Signal-to-Noise Ratio (SNR) $(\mathrm{SNR}=15 \mathrm{~dB}$ and $\mathrm{SNR}=10 \mathrm{~dB})$. For each combination of $L, M$ and SNR, the algorithms were evaluated for 50 realizations of additive noise. The RIRs were generated using the image method [15] for a shoebox-shaped room of dimensions $5 \mathrm{~m} \times 6 \mathrm{~m} \times 3 \mathrm{~m}$ and a reverberation time $T_{60}=0.5 \mathrm{~s}$. The lengths $\mathrm{L}$ were then fixed by truncating the obtained RIRs to the desired length. The input signal was a white Gaussian noise with a duration of $600 \mathrm{~s}$, which is uncorrelated with the additive noise.

For the BURQ and BNRQ algorithms, a sliding rectangular window of length $B=2 L$, overlapping by $n_{\text {off }}=L$ samples, was used. The different step-sizes were empirically optimized for each SNR such that no misconverge could be observed for $20 \mathrm{~s}$ of input signal while displaying the highest initial convergence rate. The performance of the SURQ algorithm is not shown here as it had stability issues. 
The BSI accuracy was evaluated using the scale-independent Normalized Projection Misalignment (NPM) [1] computed as follows:

$$
\operatorname{NPM}(\hat{\boldsymbol{h}}, \boldsymbol{h})=10 \log _{10}\left(\frac{\left\|\boldsymbol{h}-\frac{\hat{\boldsymbol{h}}^{T} \boldsymbol{h}}{\hat{\boldsymbol{h}}^{T} \hat{\boldsymbol{h}}}\right\|_{2}^{2}}{\boldsymbol{h}^{T} \boldsymbol{h}}\right)(\mathrm{dB}) .
$$

\subsection{Results}

Figures 1 and 2 show box plots of the mean NPMs for $M=2$ and $M=5$, where only the samples after $100 \mathrm{~s}$ were taken into account (when the algorithms were assumed to have converged). Unlike the MCLMS, NMCFLMS and BNRQ algorithms, which seem to have misconverged, the RNMCFLMS algorithm seems to have converged in NPM as the interquartile range as well as the spread in NPMs are less than $1 \mathrm{~dB}$. The BURQ algorithm consistently achieves lower NPMs on average.

By comparing the box plots for a fixed SNR and different lengths $L$ in Figures 11 and 2 , the NPMs increase as $L$ increases. This is due to the fact that near-common zeros are more likely for larger values of $L$ and fixed $M$. By comparing the box plots for fixed SNR and $L$ but different $M$ in Figures 1 and Figure 2, the NPMs decreases as $M$ increases. This is due to the fact that as $M$ increases, near-common zeros of the RIRs are less likely to appear.

While Figures 1 and 2 suggest that the BURQ algorithm consistently outperforms the other algorithms, NPM behavior over time, including the initial convergence rate, cannot be analyzed from these figures. Figure 3 provides a more detailed analysis for SNR $=15 \mathrm{~dB}, L=500, M=5$ by displaying the NPMs against time for the considered algorithms. The solid lines represent the mean NPMs, while the dotted lines show the $1^{\text {st }}$ and $9^{\text {th }}$ percentile. From this figure it can be observed that, although the MCLMS algorithm has the highest initial convergence rate, it clearly misconverges. In addition, also the NMCFLMS and the BNRQ algorithms misconverge, whereas the RNMCFLMS and the BURQ algorithms converge. Although the RNMCFLMS algorithm appears to have a faster initial convergence rate than the BURQ algorithm, the BURQ algorithm clearly outperforms the RNMCFLMS algorithm as the NPMs keep decreasing as time increases. For the other combinations of considered SNR, $L$ and $M$, a similar behavior of the NPMs has been observed.

\section{CONCLUSION}

In this paper, we have proposed novel adaptive BSI algorithms, which are based on a Rayleigh quotient cost function. Different variants have been proposed. either sample or block-based, with or without a unit-norm constraint on the stacked RIR vector. Through simulations, the unconstrained optimization algorithm, referred as BURQ, has shown to be noise-robust to additive spatially white Gaussian noise and to
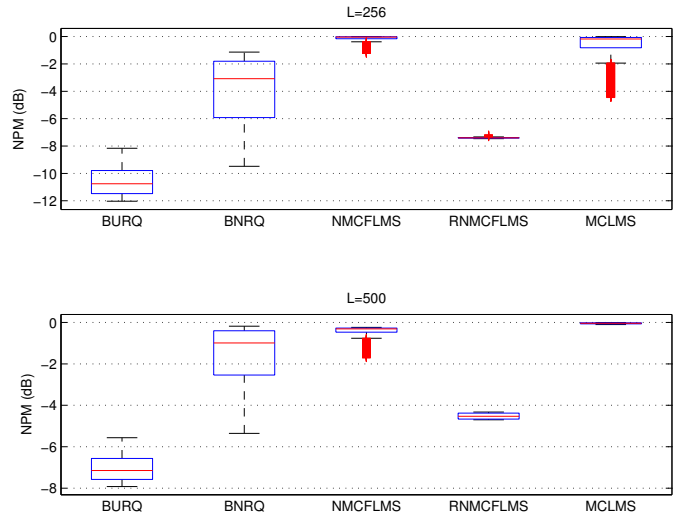

(a) $\mathrm{SNR}=15 \mathrm{~dB}$
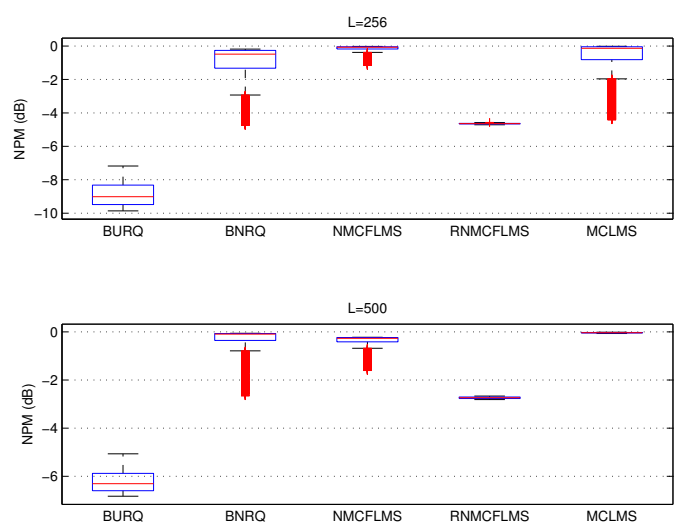

(b) $\mathrm{SNR}=10 \mathrm{~dB}$

Fig. 1: Box plot of the mean NPMs for $t>100 \mathrm{~s}$ and $M=2$

outperform the RNMCFLMS algorithm. Moreover, the NPM consistently decreases with time while the RNMCFLMS seems, at best, to achieve some limit and at worse to have an initial convergence followed by misconvergence.

\section{REFERENCES}

[1] P. A. Naylor and N. D. Gaubitch, Eds., Speech Dereverberation, Springer, 2010.

[2] M. Miyoshi and Y. Kaneda, "Inverse filtering of room acoustics," IEEE Trans. Acoust., Speech, Signal Process., vol. 36, no. 2, pp. 145-152, Feb. 1988.

[3] I. Kodrasi, S. Goetze, and S. Doclo, "Regularization for partial multichannel equalization for speech dereverberation," IEEE Trans. Audio, Speech, Lang. Process., vol. 21, no. 9, pp. 1879-1890, Sept. 2013.

[4] F. Lim, Wancheng Zhang, E.A.P. Habets, and P.A. Naylor, "Robust multichannel dereverberation using relaxed multichannel least squares," Audio, Speech, and 

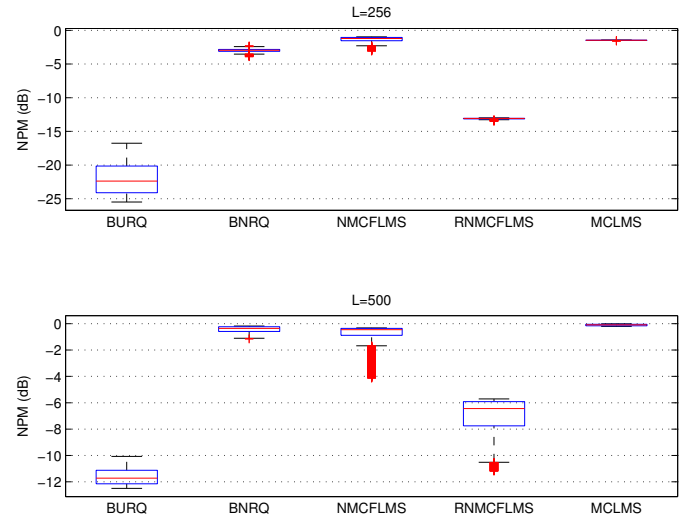

(a) $\mathrm{SNR}=15 \mathrm{~dB}$
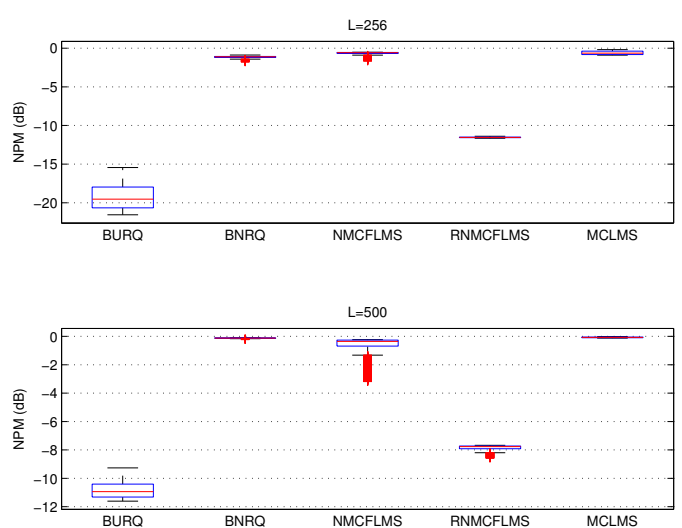

(b) $\mathrm{SNR}=10 \mathrm{~dB}$

Fig. 2: Box plots of the mean NPMs for $t>100 \mathrm{~s}$ and $M=5$

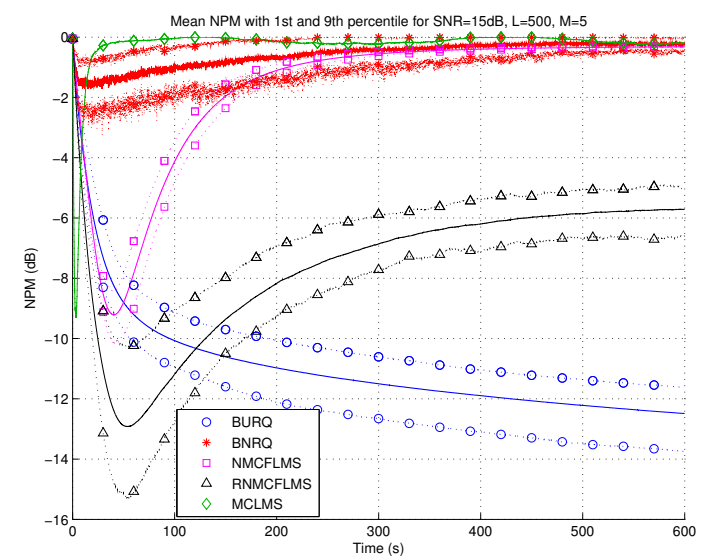

Fig. 3: Mean NPMs for each algorithm for $\mathrm{SNR}=15 \mathrm{~dB}, L=$ $500, M=5$ with the $1^{\text {st }}$ and $9^{\text {th }}$ percentile (shown in dotted lines)
Language Processing, IEEE/ACM Transactions on, vol. 22, no. 9, pp. 1379-1390, Sept 2014.

[5] Y. Huang and J. Benesty, "Adaptive multi-channel least mean square and Newton algorithms for blind channel identification," Signal Processing, vol. 82, no. 8, pp. 1127-1138, Aug. 2002.

[6] Y. Huang and J. Benesty, "A class of frequency-domain adaptive approaches to blind multichannel identification," IEEE Trans. Signal Process., vol. 51, no. 1, pp. 11-24, Jan. 2003.

[7] M. K. Hasan, N. M. Hossain, and P. A. Naylor, "Autocorrelation model-based identification method for ARMA systems in noise," IEE Proc. Vision Image Signal Processing, vol. 152, no. 5, pp. 520 - 526, 2005.

[8] N. D. Gaubitch, D. B. Ward, and P. A. Naylor, "Statistical analysis of the autoregressive modeling of reverberant speech," J. Acoust. Soc. Am., vol. 120, no. 6, pp. 4031-4039, Dec. 2006.

[9] M. A. Haque and M. K. Hasan, "Noise robust multichannel frequency-domain LMS algorithms for blind channel identification," IEEE Signal Process. Lett., vol. 15, pp. 305-308, 2008.

[10] M. R. P. Thomas, N. D. Gaubitch, E. A. P. Habets, and P. A. Naylor, "Supervised identification and removal of common filter components in adaptive blind SIMO system identification," in Proc. Intl. Workshop Acoust. Echo Noise Control (IWAENC), Tel Aviv, Israel, Aug. 2010.

[11] M. K. Hasan, J. Benesty, P. A. Naylor, and D. B. Ward, "Improving robustness of blind adaptive multichannel identification algorithms using constraints," in Proc. European Signal Processing Conf. (EUSIPCO), 2005.

[12] N. D. Gaubitch, K. Hasan, and P. A. Naylor, "Noise robust adaptive blind channel identification using spectral constraints," in Proc. IEEE Intl. Conf. on Acoustics, Speech and Signal Processing (ICASSP), Toulouse, France, May 2006, vol. 5.

[13] G. Xu, H. Liu, L. Tong, and T. Kailath, “A least-squares approach to blind channel identification," IEEE Trans. Signal Process., vol. 43, no. 12, pp. 2982-2993, Dec. 1995.

[14] M. K. Hasan and P. A. Naylor, "Analyzing effect of noise on LMS-type approaches to blind estimation of SIMO channels: robustness issue," in Proc. European Signal Processing Conf. (EUSIPCO), Florence, Italy, Sept. 2006.

[15] J. B. Allen and D. A. Berkley, "Image method for efficiently simulating small-room acoustics," J. Acoust. Soc. Am., vol. 65, no. 4, pp. 943-950, Apr. 1979. 